typeset using JPSJ.sty $<$ ver.1.0b $>$

\title{
Electronic states in correlated three-coupled chains
}

\author{
M. Tsuchiizu ${ }^{a}$, Y. Suzumura ${ }^{a, b}$ \\ ${ }^{a}$ Department of Physics, Nagoya University, Nagoya 464-8602, Japan \\ ${ }^{b}$ CREST, Japan Science and Technology Corporation (JST)
}

(Received

)

\begin{abstract}
Three-coupled chains of quarter-filled Hubbard model with dimerization have been studied for periodic boundary conditions. By use of a renormalization group method, it is found that the interchain hopping is renormalized to zero leading to confinement when charge gap becomes larger than the effective interchain hopping energy. Such a result of confinement is compared with that of two-coupled chains.
\end{abstract}

KEYWORDS: A. organic compounds, D. electronic structure, D. phase transition

Spin gap and charge gap have been studied extensively in coupled-chain systems. Theoretical study in terms of a renormalization group (RG) method shows the spin gap in two-coupled chains for small but relevant interchain hopping [1] where the electronic state is given by $d_{x^{2}-y^{2}}$ like superconducting state. The spin gap has been also examined for three-coupled chains [2, 3, 4], whose state depends on boundary conditions in the transverse direction. In case of small interchain hopping at half-filling, there are both charge gap and spin gap for open boundary conditions (OBC) while charge gap vanishes for periodic boundary conditions (PBC). A system with infinite chains at half-filling exhibits charge gap but no spin gap [5].

Although these works treat the relevant interchain hopping, a theory also suggests the irrelevant one-particle interchain hopping for half-filling $[5]$. In order to understand optical experiments on quasi-one-dimensional organic conductors [6, 7] indicating a confinement - deconfinement transition, two-coupled chains at half-filling have been studied where a transition from deconfinement to confinement occurs for umklapp scattering being larger than a critical value [8,9]. In the present paper, such a transition is examined for three-coupled chains. Although the results of two chains seem to explain the experiments, it is not yet known if such a transition can be expected for many chains.

The Hamiltonian for quarter-filled three-coupled chains with on-site repulsion $(U)$ and interchain hopping $\left(t_{\perp}\right)$ is written as

$$
\begin{aligned}
\mathcal{H}= & -\sum_{j, \sigma, l}\left[t+(-1)^{j} t_{\mathrm{d}}\right]\left(c_{j \sigma l}^{\dagger} c_{j+1 \sigma l}+\text { h.c. }\right) \\
& -t_{\perp} \sum_{j, \sigma, l}\left(c_{j \sigma l}^{\dagger} c_{j \sigma l+1}+\text { h.c. }\right)+U \sum_{j, l} n_{j \uparrow l} n_{j \downarrow l}(1)
\end{aligned}
$$

where $n_{j \sigma l}=c_{j \sigma l}^{\dagger} c_{j \sigma l}$ and $c_{j \sigma l}$ denotes the annihilation operator of the electron at the $j$-th site of the $l$-th chain $(l=1,2,3)$ with spin $\sigma(=\uparrow, \downarrow)$, and $c_{j \sigma 4}=c_{j \sigma 1}$. In Eq. (1), $t_{\mathrm{d}}$ denotes dimerization along the chains and the case for PBC is studied.
Diagonalizing the $t_{\mathrm{d}}$-term, which leads effectively to the half-filled band [9], we consider an effective Hamiltonian $\mathcal{H}^{d}$ consisting of the lower band with fermion operators of $d_{k \sigma l}$. The terms for the interchain hopping can be diagonalized by introducing Fourier transform, $a_{k \sigma \mu} \equiv$ $(1 / \sqrt{3}) \sum_{l=1}^{3} \exp \left[-i k_{y}(\mu) l\right] d_{k \sigma l}$ with $k_{y}(\mu)=(2 \pi / 3) \mu$ $(\mu=0, \pm 1)$. The kinetic term is written as $\mathcal{H}_{K}^{d} \equiv \sum_{k, \sigma, \mu}$ $\varepsilon\left(k, k_{y}\right) a_{k \sigma \mu}^{\dagger} a_{k \sigma \mu}$ with $\varepsilon\left(k, k_{y}\right)=-2 \sqrt{t^{2} \cos ^{2} k a+t_{\mathrm{d}}^{2} \sin ^{2} k a}-$ $2 t_{\perp} \cos k_{y}$, which is rewritten, in terms of the linearized dispersion, as $\mathcal{H}_{K}^{d}=\sum_{k, p, \sigma, \mu} v_{\mathrm{F}}\left(p k-k_{\mathrm{F} \mu}\right) a_{k p \sigma \mu}^{\dagger} a_{k p \sigma \mu}$ with $p$ being the index of the branch $p=+(-)$ corresponding to right moving (left moving) electrons. Fermi momenta are given by $k_{\mathrm{F} 0}=k_{\mathrm{F}}+2 t_{\perp} / v_{\mathrm{F}}$ and $k_{\mathrm{F} \pm}=$ $k_{\mathrm{F}}-t_{\perp} / v_{\mathrm{F}}$ where $v_{\mathrm{F}}=\sqrt{2} t a\left[1-\left(t_{\mathrm{d}} / t\right)^{2}\right] / \sqrt{1+\left(t_{\mathrm{d}} / t\right)^{2}}$ [9]. Following the conventional $g$-ology, coupling constants are given by $g_{1 \perp}=g_{2} \perp=g_{4^{\perp}}=U a, g_{3}=$ $U a\left(2 t_{\mathrm{d}} / t\right) /\left[1+\left(t_{\mathrm{d}} / t\right)^{2}\right]$ [9] and $g_{1 \|}=g_{2} \|=g_{4 \|}=0$.

Applying the bosonization method, we introduce phase variables $\theta_{\rho \mu}$ and $\theta_{\sigma \mu}$ expressing fluctuations of the charge density and spin density for the $\mu$-band [10], where the conjugate phase is introduced by $\left[\theta_{\nu \mu}(x), \phi_{\nu^{\prime} \mu^{\prime}}\left(x^{\prime}\right)\right]_{-}=$ $i \pi \delta_{\nu, \nu^{\prime}} \delta_{\mu, \mu^{\prime}} \operatorname{sgn}\left(x-x^{\prime}\right)\left(\nu, \nu^{\prime}=\rho, \sigma\right)$. Thus we obtain the total Hamiltonian given by $\mathcal{H}^{d}=\mathcal{H}_{0}+\mathcal{H}_{I}$ where $\mathcal{H}_{0}$ expresses bilinear terms of density operators, and $\mathcal{H}_{I}$ denotes nonlinear terms. Here we define the new phase variables as $Y_{1} \equiv\left(X_{1}+X_{2}+X_{3}\right) / \sqrt{3}, Y_{2} \equiv\left(X_{1}-X_{3}\right) / \sqrt{2}$ and $Y_{3} \equiv\left(-X_{1}+2 X_{2}-X_{3}\right) / \sqrt{6}$ where $\left(Y_{1}, Y_{2}, Y_{3}\right)=$ $\left(\theta_{\rho}, \theta_{\mathrm{C} 1}, \theta_{\mathrm{C} 2}\right)$ and $\left(X_{1}, X_{2}, X_{3}\right)=\left(\theta_{\rho+}, \theta_{\rho 0}, \theta_{\rho-}\right)$ and the same transformation is applied to $\rho \rightarrow \sigma$ and also to the conjugate variables $\phi_{\nu \mu}$. Thus $\mathcal{H}_{0}$ is rewritten as $\mathcal{H}_{0}=\sum_{\nu}\left(v_{\nu} / 4 \pi\right) \int d x\left[K_{\nu}^{-1}\left(\partial \theta_{\nu}\right)^{2}+K_{\nu}\left(\partial \phi_{\nu}\right)^{2}\right]$ with $\nu=$ $\rho, \mathrm{C} 1, \mathrm{C} 2, \sigma, \mathrm{S} 1, \mathrm{~S} 2$, where $v_{\rho(\sigma)}=v_{\mathrm{F}}\left[1+(-) U / \pi v_{\mathrm{F}}\right]^{1 / 2}$, $K_{\rho(\sigma)}=\left[1+(-) U a / \pi v_{\mathrm{F}}\right]^{-1 / 2}, v_{\mathrm{C} 1}=v_{\mathrm{S} 1}=v_{\mathrm{C} 2}=v_{\mathrm{S} 2}=$ $v_{\mathrm{F}}$ and $K_{\mathrm{C} 1}=K_{\mathrm{S} 1}=K_{\mathrm{C} 2}=K_{\mathrm{S} 2}=1$. The Hamiltonian, $\mathcal{H}_{I}$ is divided as $\mathcal{H}_{I}=\mathcal{H}_{1}+\mathcal{H}_{3}+\mathcal{H}_{2}+\mathcal{H}_{\|}$where the respective term is written as $(i=1,3,2, \|)$

$$
\mathcal{H}_{i}=\sum_{Z} \frac{v_{\mathrm{F}}}{\pi \alpha^{2}} G_{i Z} \int d x \cos \Theta_{i Z} \cdot h_{i Z},
$$

$(Z=A \simeq Z)$ and $\alpha$ is a cutoff of the order of lat- 


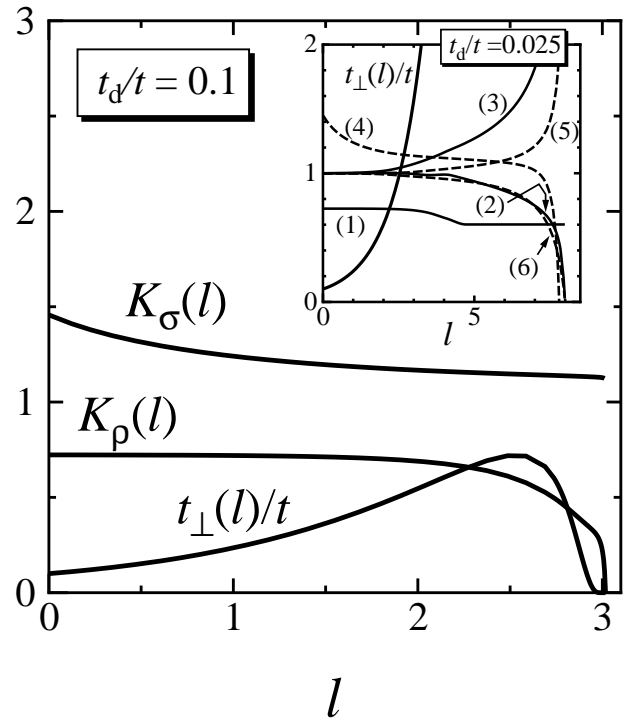

Fig. 1. Quantities $t_{\perp}(l) / t, K_{\rho}(l)$ and $K_{\sigma}(l)$ as a function of $l$ for $U / t=4, t_{\perp} / t=0.1$ and $t_{\mathrm{d}} / t=0.1$. The inset shows $t_{\perp}(l) / t$, $K_{\rho}(1), K_{\mathrm{C} 1}(2), K_{\mathrm{C} 2}(3), K_{\sigma}(4), K_{\mathrm{S} 1}(5)$ and $K_{\mathrm{S} 2}(6)$ for $U / t=4$, $t_{\perp} / t=0.1$ and $t_{\mathrm{d}} / t=0.025$.

tice constant and $h_{i Z}$ denotes the product of the Majorana fermion operators introduced to retain the anticommutation relation of the field operators [3]. The phase variables of the backward scattering term with opposite spins, $\mathcal{H}_{1}$, are given by $\Theta_{1 Z}=(2 / \sqrt{3}) \theta_{\sigma}+\widetilde{\Theta}_{1 Z}$ where $\widetilde{\Theta}_{1 A}=\epsilon \sqrt{2} \theta_{\mathrm{S} 1}-\sqrt{2 / 3} \theta_{\mathrm{S} 2}, \widetilde{\Theta}_{1 B}=2 \sqrt{2 / 3} \theta_{\mathrm{S} 2}, \widetilde{\Theta}_{1 C}=$ $\left[\left(\epsilon^{\prime} \theta_{\mathrm{S} 1}+\theta_{\mathrm{S} 2} / \sqrt{3}\right)+\epsilon\left(\theta_{\mathrm{C} 1}-\epsilon^{\prime} \sqrt{3}\left(\theta_{\mathrm{C} 2}+2 \sqrt{6} t_{\perp} x / v_{\mathrm{F}}\right)\right)\right] / \sqrt{2}$, $\widetilde{\Theta}_{1 D}=\epsilon \sqrt{2} \theta_{\mathrm{C} 1}-\sqrt{2 / 3} \theta_{\mathrm{S} 2}, \widetilde{\Theta}_{1 E}=\left[\left(\epsilon^{\prime} \theta_{\mathrm{S} 1}+\theta_{\mathrm{S} 2} / \sqrt{3}\right)+\right.$ $\left.\epsilon\left(\phi_{\mathrm{S} 1}-\epsilon^{\prime} \sqrt{3} \phi_{\mathrm{S} 2}\right)\right] / \sqrt{2}, \widetilde{\Theta}_{1 F}=\epsilon \sqrt{2} \phi_{\mathrm{S} 1}-\sqrt{2 / 3} \theta_{\mathrm{S} 2}, \widetilde{\Theta}_{1 G}=$ $\left(\theta_{\mathrm{S} 2}+\epsilon^{\prime} \sqrt{3} \theta_{\mathrm{C} 1}+\epsilon 3 \phi_{\mathrm{C} 2}-\epsilon \epsilon^{\prime} \sqrt{3} \phi_{\mathrm{S} 1}\right) / \sqrt{6}$ and $\widetilde{\Theta}_{1 H}=\left[\left(\epsilon^{\prime \prime} \theta_{\mathrm{S} 1}-\right.\right.$ $\left.\theta_{\mathrm{S} 2} / \sqrt{3}\right)+\epsilon^{\prime}\left(\theta_{\mathrm{C} 1}+\epsilon^{\prime \prime} \sqrt{3}\left(\theta_{\mathrm{C} 2}+2 \sqrt{6} t_{\perp} x / v_{\mathrm{F}}\right)\right)+\epsilon\left(\epsilon^{\prime \prime} 3 \phi_{\mathrm{C} 1}-\right.$ $\left.\left.\sqrt{3} \phi_{\mathrm{C} 2}\right)-\epsilon \epsilon^{\prime}\left(\phi_{\mathrm{S} 1}+\epsilon^{\prime \prime} \sqrt{3} \phi_{\mathrm{S} 2}\right)\right] / 2 \sqrt{2}$. In Eq. (2), the sum is taken implicitly with respect to $\epsilon, \epsilon^{\prime}$ and $\epsilon^{\prime \prime}(= \pm)$ which lead to the distinction for $h_{i Z}$ but not for $G_{i Z}$. The umklapp scattering terms, $\mathcal{H}_{3}$, are obtained from $\mathcal{H}_{1}$ by replacing $G_{1 Z} \rightarrow G_{3 Z}$ and $\left(\theta_{\sigma}, \theta_{\mathrm{S} 1}, \theta_{\mathrm{S} 2}\right) \leftrightarrow\left(\theta_{\rho}, \theta_{\mathrm{C} 1}, \theta_{\mathrm{C} 2}+\right.$ $\left.2 \sqrt{6} t_{\perp} x / v_{\mathrm{F}}\right)$. The phase variables of the forward scattering term with opposite spins, $\mathcal{H}_{2}$, are expressed as $\Theta_{2 E}=$ $\left[\left(\theta_{\mathrm{C} 1}-\epsilon^{\prime} \sqrt{3}\left(\theta_{\mathrm{C} 2}+2 \sqrt{6} t_{\perp} x / v_{\mathrm{F}}\right)\right)+\epsilon\left(\phi_{\mathrm{S} 1}-\epsilon^{\prime} \sqrt{3} \phi_{\mathrm{S} 2}\right)\right] / \sqrt{2}$, $\Theta_{2 F}=\sqrt{2} \theta_{\mathrm{C} 1}+\epsilon \sqrt{2} \phi_{\mathrm{S} 1}, \Theta_{2 G}=\left(\theta_{\mathrm{C} 1}-\epsilon^{\prime} \sqrt{3} \theta_{\mathrm{S} 2}-\epsilon \epsilon^{\prime} \sqrt{3} \phi_{\mathrm{C} 2}+\right.$ $\left.\epsilon \phi_{\mathrm{S} 1}\right) / \sqrt{2}$ and $\Theta_{2 H}=\left[\left(\theta_{\mathrm{C} 1}+\epsilon^{\prime \prime} \sqrt{3}\left(\theta_{\mathrm{C} 2}+2 \sqrt{6} t_{\perp} x / v_{\mathrm{F}}\right)\right)-\right.$ $\left.\epsilon^{\prime}\left(\epsilon^{\prime \prime} 3 \theta_{\mathrm{S} 1}-\sqrt{3} \theta_{\mathrm{S} 2}\right)-\epsilon \epsilon^{\prime}\left(\epsilon^{\prime \prime} 3 \phi_{\mathrm{C} 1}-\sqrt{3} \phi_{\mathrm{C} 2}\right)+\epsilon\left(\phi_{\mathrm{S} 1}+\epsilon^{\prime \prime} \sqrt{3} \phi_{\mathrm{S} 2}\right)\right]$ The forward scattering term with parallel spins, $\mathcal{H}_{\|}$is obtained from $\mathcal{H}_{2}$ by replacing $G_{2 Z} \rightarrow G_{\| Z}(Z=E \sim$ $H)$ and $\left(\theta_{\mathrm{S} 1}, \theta_{\mathrm{S} 2}\right) \leftrightarrow\left(\phi_{\mathrm{S} 1}, \phi_{\mathrm{S} 2}\right)$. Coupling constants are given by $G_{i A}=G_{i B}=G_{i C}=G_{i D}=G_{i E}=G_{i F}=$ $G_{i G}=G_{i H}=g_{i} / 6 \pi v_{\mathrm{F}}(i=1,3)$ and $G_{i E}=G_{i F}=$ $G_{i G}=G_{i H}=g_{i} / 6 \pi v_{\mathrm{F}}(i=2, \|)$ where $g_{\|} \equiv g_{2 \|}-g_{1 \|}$. Non-linear terms of forward scattering with the same $p$ branch are discarded because these effect is negligibly small. By assuming scaling invariance with respect to $\alpha \rightarrow \alpha^{\prime}=\alpha \mathrm{e}^{d l}$, the second order RG equation for the interchain hopping is given by



Fig. 2. Normalized quantity $t_{\perp}(l) / t$ for choices of $U / t=3, U_{c} / t$ and 4.5 with $t_{\perp} / t=0.1$ and $t_{\mathrm{d}} / t=0.1$ where $U_{c} / t \simeq 3.7$. The solid and dotted curves are explained in the text.

$$
\frac{d}{d l} \tilde{t}_{\perp}=\tilde{t}_{\perp}-F\left(\tilde{t}_{\perp},\left\{G_{i Z}\right\}\right) K_{\mathrm{C} 2},
$$

where $\tilde{t}_{\perp} \equiv t_{\perp} /\left(v_{\mathrm{F}} \alpha^{-1}\right), F\left(\tilde{t}_{\perp},\left\{G_{i Z}\right\}\right)=\left[G_{1 C}^{2}+G_{2 E}^{2}+\right.$ $\left.G_{\| E}^{2}\right] J_{1}\left(6 \tilde{t}_{\perp}\right)+\left[G_{1 H}^{2}+G_{2 H}^{2}+G_{\| H}^{2}\right] J_{1}\left(3 \tilde{t}_{\perp}\right)+\left[G_{3 A}^{2}+G_{3 D}^{2}+\right.$ $\left.G_{3 F}^{2}\right] J_{1}\left(4 \tilde{t}_{\perp}\right) / 3+\left[G_{3 C}^{2}+G_{3 E}^{2}+G_{3 G}^{2}\right] J_{1}\left(2 \tilde{t}_{\perp}\right) / 3+G_{3 B}^{2} J_{1}\left(8 \tilde{t}_{\perp}\right) / 3+$ $G_{3 H}^{2} J_{1}\left(\tilde{t}_{\perp}\right) / 3$ and $J_{n}$ is $n$-th Bessel function. Equation (3) is solved together with RG equations for $G_{i Z}$ where $l$ is related to energy scale $\omega$ or temperature $T$ by $l=\ln (W / \omega)$ or $\ln (W / T)$ with $W\left(\equiv v_{\mathrm{F}} \alpha^{-1}\right)$ being of the order of band width. We take $\alpha=2 a / \pi$ 9]. It is noted that the r.h.s. of eq. (3) for small $\tilde{t}_{\perp}$ is reduced to $\tilde{t}_{\perp}\left[1-\left(G_{1}^{2}+G_{2}^{2}+G_{\|}^{2}+G_{3}^{2}\right) / 2\right]$ which becomes the same as that of many chains [5].

In Fig. 1 , the $l$-dependence of coupling constants is shown for $t_{\mathrm{d}} / t=0.1$ and $t_{\mathrm{d}} / t=0.025$ with $U / t=4$ and $t_{\perp} / t=0.1$. For $t_{\mathrm{d}} / t=0.025$ (inset), the interchain hopping $t_{\perp}(l)$ becomes relevant corresponding to deconfinement where $K_{\rho}$ remains finite and $K_{\mathrm{C} 1}, K_{\sigma}$ and $K_{\mathrm{S} 2}\left(K_{\mathrm{C} 2}\right.$ and $\left.K_{\mathrm{S} 1}\right)$ decrease to zero (become infinite). The curves are calculated, for simplicity, by setting $J_{n}(l)=0$ for $l>l_{n}$ with $l_{n}$ corresponding the first node of the Bessel function, although such a treat$/ 2 n=2 \mathrm{~h} t$ gives negligible difference in the numerical results. When $K_{\nu}(l)$ decreases to zero or increases to infinity, the corresponding phase is locked leading to a formation of gap, where relevant coupling constants are $G_{1 B}(\rightarrow-\infty)$, $G_{1 D}(\rightarrow-\infty), G_{1 F}(\rightarrow-\infty), G_{1 G}(\rightarrow+\infty), G_{2 F}(\rightarrow-\infty)$ and $G_{2 G}(\rightarrow+\infty)$. There are two kinds of gap for charge fluctuations and three kinds of gap for spin fluctuations. For $t_{\mathrm{d}} / t=0.1$ (main figure), one finds confinement where $t_{\perp}(l)$ decreases to zero after taking a maximum. The $l$-dependence of $K_{\rho}(l)$ implies charge gap in the total charge fluctuation, which is in contrast to the case of deconfinement. For $t_{\mathrm{d}} / t=0.1$, exponents $K_{\nu}(l)(\nu=\mathrm{C} 1$, $\mathrm{C} 2, \sigma, \mathrm{S} 1, \mathrm{~S} 2)$ remain finite, i.e., $K_{\nu}(l) \simeq 1$ at $l \simeq 3$. 


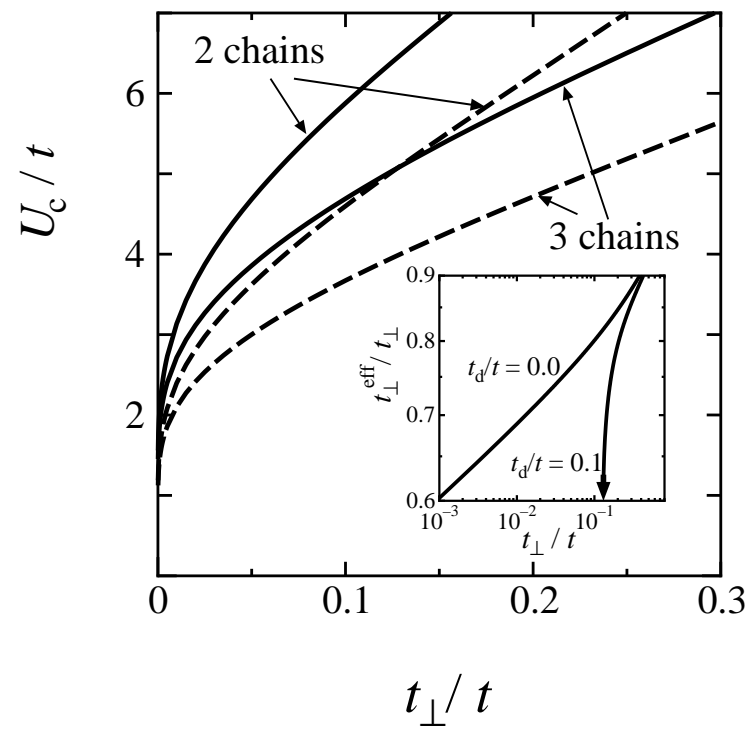

Fig. 3. The critical values $U_{c}$ as a function of $t_{\perp}$ with fixed $t_{\mathrm{d}} / t=0.05$ (solid curves) and 0.1 (dashed curves) for threecoupled chains and two-coupled chains 9 . The inset denotes the $\log t_{\perp}^{\text {eff }} / t_{\perp}-\log t_{\perp}$ plot for $t_{\mathrm{d}} / t=0.0$ and 0.1 with fixed $U / t=4.0$ where the arrow shows the critical value for the confinement.

In Fig. $2, t_{\perp}(l) / t$ is shown with the fixed $U / t=3, U_{c} / t(\simeq$ 3.7) and 4.5 where solid (dotted) curves denote $t_{\perp}(l) / t$ for $0<l<l_{\Delta}\left(l>l_{\Delta}\right)$ with $K_{\rho}\left(l_{\Delta}\right) \equiv K_{\rho} / 2$. The case for $l>l_{\Delta}$ is invalid since the magnitude of the umklapp scattering increases to infinity. In the curve $t_{\perp}(l)$, there is a maximum given by $\max \left[t_{\perp}(l) / t\right] \simeq 3.4(0.5)$ at $l \simeq 4.0(2.2)$ for $U / t=3.0(4.5)$ although the maximum for $U / t=3.0$ is located in the region of $l>l_{\Delta}$. Thus the case for $U / t=3.0$ (4.5) corresponds to deconfinement (confinement). The boundary between confinement and deconfinement is determined by the condition that $\max \left[t_{\perp}(l) / t\right] \simeq 1$ at $U=U_{c}$.

In Fig. 3, the $t_{\perp}$-dependence of $U_{c}$ is shown for $t_{\mathrm{d}} / t=$ 0.05 (solid curves) and 0.1 (dashed curves) where confinement (deconfinement) is obtained for $U>U_{c}(U<$ $\left.U_{c}\right)$. For comparison, the corresponding results for twocoupled chains [9] are also shown. The critical values for three-coupled chains is smaller than that for twocoupled chains. Since the RG equation of two-coupled chains corresponding to Eq. (3) includes the Bessel function with only $J_{1}\left(8 \tilde{t}_{\perp}\right)$, the effect of umklapp scattering for three-coupled chains is stronger than that for twocoupled chains. The effective interchain hopping $t_{\perp}^{\text {eff }}$ can be evaluated from $t_{\perp}^{\text {eff }}=t \exp \left[-l_{\text {eff }}\right]$ where $t_{\perp}\left(l_{\text {eff }}\right) / t=1$ [11. In the inset, $t_{\perp}^{\mathrm{eff}} / t_{\perp}$ is shown as a function of $t_{\perp}$ on a logarithmic scale. The power-law behavior of $t_{\perp}^{\text {eff, } 0}\left(\equiv t_{\perp}^{\text {eff }}\left(g_{3}=0\right)\right)$ for small $t_{\perp}$ is consistent with the analytical formula $t_{\perp}^{\text {eff, } 0} \simeq t_{\perp}\left(t_{\perp} / W\right)^{\alpha_{0} /\left(1-\alpha_{0}\right)}$ with $\alpha_{0}=\left(K_{\rho}+K_{\rho}^{-1}+K_{\sigma}+K_{\sigma}^{-1}-4\right) / 4$ [5]. In the presence of the dimerization, $t_{\perp}^{\text {eff }}$ is reduced from that of the power-law behavior and has a critical value below which the confinement occurs.

The confinement-deconfinement transition is examined

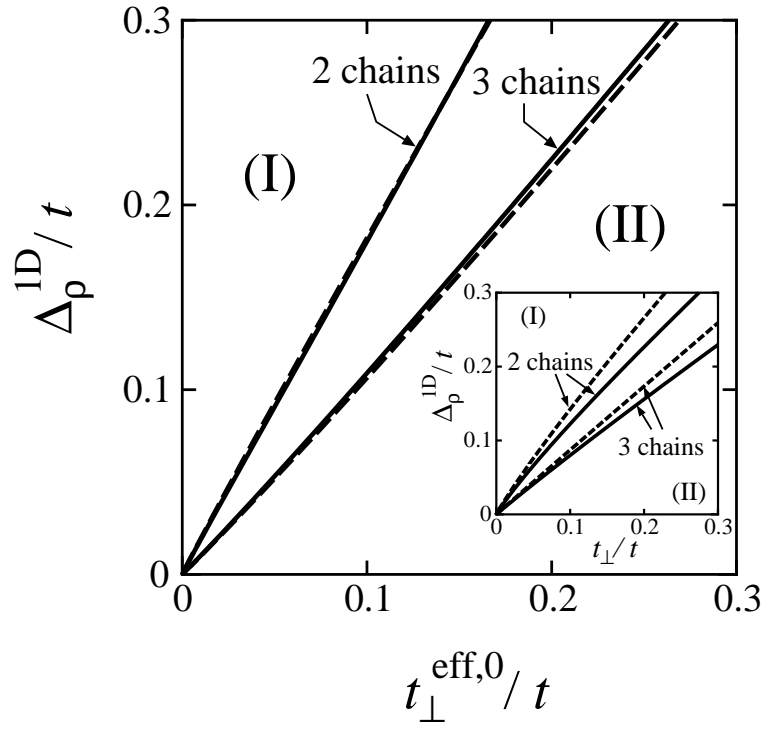

Fig. 4. The phase diagram of confinement (I) and deconfinement (II) in case of $t_{\mathrm{d}} / t=0.05$ (solid line) and 0.1 (dashed line) on a plane of the effective interchain hopping $t_{\perp}^{\text {eff, } 0}$ and $\Delta_{\rho}^{1 \mathrm{D}}$. The inset denotes the corresponding phase diagram on the plane of $t_{\perp}$ and $\Delta_{\rho}^{1 \mathrm{D}}$.

in terms of the charge gap induced by the umklapp scattering. The charge gap for the single chain is obtained as the function of $U$ and $t_{\mathrm{d}}$ by a method $\Delta_{\rho}^{1 \mathrm{D}}=W \exp \left[-l_{\Delta}\right]$ with $K_{\rho}\left(l_{\Delta}\right)=K_{\rho} / 2$ for $t_{\perp}=0$. Such gap has a meaning of a characteristic energy of the umklapp scattering even for the deconfined region in which the charge gap is reduced to zero due to the presence of the misfit for all the nonlinear terms of umklapp scattering. In the inset of Fig. 4, a phase diagram of confinement (I) and deconfinement (II) is shown on the plane of the bare interchain hopping $t_{\perp}$ and $\Delta_{\rho}^{1 \mathrm{D}}$ where the boundary for two-coupled chains [9] are also shown for comparison. Although the ratio of $\Delta_{\rho}^{1 \mathrm{D}} / t_{\perp}$ is nearly constant, the curve is slightly convex upward for small $t_{\perp}$. In the main figure of Fig. 4, the phase diagram with the same parameter is shown on the plane of $t_{\perp}^{\mathrm{eff}, 0} / t$ and $\Delta_{\rho}^{1 \mathrm{D}} / t$. The quantity $t_{\perp}^{\mathrm{eff}, 0}$ denotes the effective interchain hopping, which is renormalized by the intrachain interaction without umklapp scattering. The ratio of $\Delta_{\rho}^{1 \mathrm{D}}$ to $t_{\perp}^{\mathrm{eff}, 0}$ at the boundary is estimated as follows when $0.05<t_{\perp} / t<0.3$. The ratio for three-coupled chains is given by $1.0 \lesssim \Delta_{\rho}^{1 \mathrm{D}} / t_{\perp}^{\mathrm{eff}, 0} \lesssim 1.1$ while that for two-coupled chains is given by $1.8 \lesssim \Delta_{\rho}^{1 \mathrm{D}} / t_{\perp}^{\text {eff, } 0} \lesssim 1.9$. By noting that the $t_{\mathrm{d}}$-dependence of the boundary is very small, it turns out that the confinement-deconfinement transition is determined by the competition between the charge gap and the effective interchain hopping energy.

We briefly discuss the case for OBC where the RG equation for the interchain hopping takes more complicated form due to twelve coupling constants for umklapp scattering. The small difference between PBC and OBC is expected since the RG equation for the interchain hopping has the same limiting form as that of PBC for small 
$\tilde{t}_{\perp}$. Actually, we find almost the same boundary as Fig. 3 when the solution of single chain is substituted for the RG equation of interchain hopping.

In summary, the confinement-deconfinement transition in the three-coupled Hubbard chains with dimerization has been shown for PBC when the effective interchain hopping energy becomes of the order the charge gap induced by the umklapp scattering.

The authors thank H. Yoshioka for useful discussions. This work was supported by a Grant-in-Aid for Scientific Research from the Ministry of Education, Science, Sports and Culture (Grant No.09640429), Japan.

[1] D.V. Khveshchenko, T.M. Rice, Phys. Rev. B 50 (1994) 252 and references therein.

[2] E. Arrigoni, Phys. Status Solidi B 195 (1996) 425, Phys. Lett. A 215 (1996) 91.

[3] H. J. Schulz, cond-mat/9605075

[4] T. Kimura, K. Kuroki, H. Aoki, Phys. Rev. B 54 (1996) R9608, J. Phys. Soc. Jpn. 67 (1998) 1377.

[5] C. Bourbonnais, in Strongly Interacting Fermions and High $T_{c}$ Superconductivity, B. Douçot and J. Zinn-Justin, Eds. (Elsevier, Amsterdam, 1995), p. 307.

[6] V. Vescoli et al., Science 281 (1998) 1181.

[7] A. Schwartz et al., Phys. Rev. B 58 (1998) 1261.

[8] Y. Suzumura, M. Tsuchiizu, G. Grüner, Phys. Rev. B 57 (1998) R15040.

[9] M. Tsuchiizu, Y. Suzumura, Proc. Int. Conf. SCES '99, Nagano 1999, to be published in Physica B,

[10] Y. Suzumura, Prog. Theor. Phys. 61 (1979) 1.

[11] M. Tsuchiizu, Y. Suzumura, T. Giamarchi, Prog. Theor. Phys. 101 (1999) 763. 